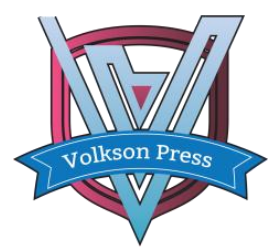

Contents List available at VOLKSON PRESS

Mechanical and Control Engineering (MCE)

DOI : http://doi.org/10.26480/wsmce.01.2017.76.78

\title{
RESEARCH ON ADAPTIVE SLIDING MODE CONTROL FOR PIEZOELECTRIC POSITIONING SYSTEM
}

\author{
Yang Gui-hua ${ }^{1 *}$, Zhong Zhi-xian ${ }^{1}$, Liang $\mathbf{Y u}^{2}$ \\ ${ }^{1}$ College of Mechanical and Control Engineering, Guilin University of Technology, Guilin 541004, China. \\ ${ }^{2}$ Bowen College of Management, Guilin University of Technology, Guilin 541004, China \\ *Corresponding Author Email: 954991219@qq.com
}

This is an open access article distributed under the Creative Commons Attribution License, which permits unrestricted use, distribution, and reproduction in any medium, provided the original work is properly cited

\section{ARTICLE DETAILS}

\section{Article History:}

Received 02 october 2017

Accepted 06 october 2017

Available online 11 november 2017

Keywords

sliding mode control, piezoelectric

positioning system, adaptive

control, chattering

\section{ABSTRACT}

It is difficult to obtain the accurate mathematical model of the controlled object for the hysteresis nonlinearity of the piezoelectric actuator. An adaptive sliding mode control strategy based on switching gain and boundary layer thickness is proposed. The chattering phenomenon is reduced, and the tracking accuracy is guaranteed, and it has good robustness to external disturbance. The stability of the whole closed-loop system is proved by the Lyapunov criterion, and the control method is simple and reliable. Simulation results show the effectiveness of the proposed algorithm.

\section{Introduction}

Ceramic piezoelectric actuator, with its strong actuating force, capability of high resolving power, high frequency response, compactness and other advantages, has been widely applied to the field of precision positioning. However, the inherent hysteresis nonlinearity of its smart material substantially reduces the control precision of positioning system, and may also causes systematic divergence [1]. The hysteresis nonlinearity of the piezoelectric actuator makes it hard to capture an accurate mathematic model of the controlled object, and the parameter variation and external disturbance in the course of running also limits the improvement of precision positioning. Therefore, one should not only have a nominal design and but consider the uncertainties which may have an impact on stability and other performances when designing for an uncertain nonlinear system. This research introduces a combined adaptive and sliding mode control strategy. By applying adaptive control to sliding control, the methodology exploits both advantages to reduce the reliance on precision of control model. This should be theoretically and practically significant for control designing for nonlinear system under uncertain condition.

\section{MATHEMATIC MODEL FOR HYSTERESIS NONLINEARITY OF PIEZOELECTRIC ACTUATOR}

A typical ceramic actuator mainly consists of three parts: piezoelectric stack, preloaded spring and output displacement slider. The hysteresis nonlinearity of the piezoelectric ceramic material is referred to as a nonlinear relationship between input voltage and output displacement, i.e. when one applies a voltage to the piezoelectric ceramic actuator, the curve, which shows the increase and decrease of output displacement and input voltage, presents an asymmetric hysteresis, causing positioning error of the movement mechanism [2].

Currently, some modeling approaches for hysteretic system, such as Preisach model, Winer model, genetic algorithm and artificial neural network, are used to reduce hysteresis of piezoelectric positioning system [3-5]. The dynamic characteristic of piezoelectric stage is a manifestation of the piezoelectric drive amplifier, piezoelectric actuator and the flexible hinge support together. A dynamic hysteresis model for piezoelectric stage introduced by a group of researcher a comprehensive characterization of its dynamic characteristic [6]. When working frequency of piezoelectric stage is far lower than resonant frequency, (as is often the case), distributing parameter of the piezoelectric stage equals to quality-springdamp system described in Figure 1.

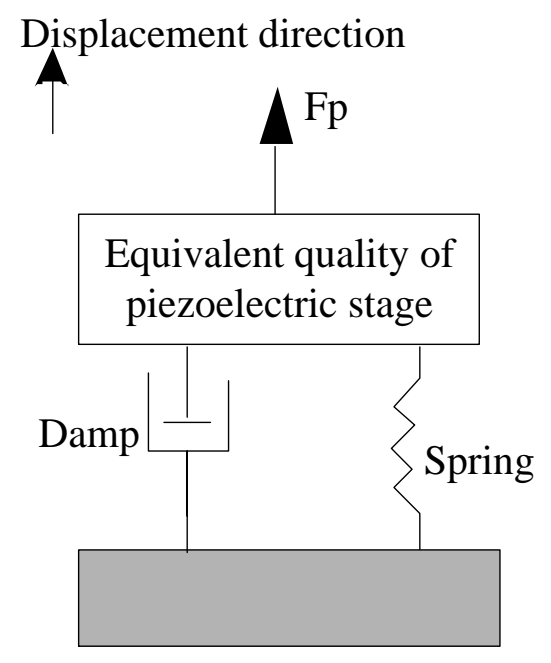

Figure 1: Equivalent model of piezoelectric stage

Figure 1 shows the force analysis of the equivalent model of the whole piezoelectric stage. Using Newton's law of motion, the force analysis can be defined as follows:

$$
m \ddot{y}(t)+f \ddot{y}(t)+k y(t)=F_{P}(t)(1)
$$

where $m, f, k, y(t), F_{p}(t)$ denote equivalent quality, equivalent damping ratio, spring constant, micro-displacement output and piezoelectric stack internal stress respectively. And input voltage is a hysteretic nonlinear relationship defined as

$$
F_{P}(t)=H[u(t)] \quad(2)
$$


Set $\xi=\frac{f}{2 \sqrt{k m}}, \omega_{n}=\sqrt{\frac{k}{m}}, H^{*}[u(t)]=\frac{H[u(t)]}{\omega_{n}{ }^{2} m}$

then equation (1) can be defined as:

$$
\ddot{y}(t)+2 \xi \omega_{n} \dot{y}(t)+\omega_{n}{ }^{2} y(t)=\omega_{n}{ }^{2} H^{*}[u(t)](3)
$$

According to Equation (3), the model structure of the piezoelectric stage can be viewed as a cascade of a nonlinear hysteresis $H^{*}$ and a linear second-order system.

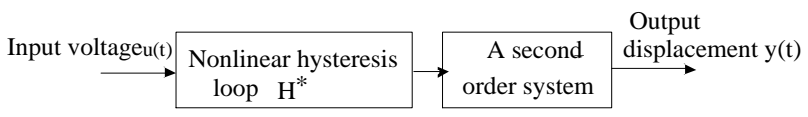

Figure 2: Equivalent model structure of piezoelectric stage

\section{CONTROL SYSTEM OF PIEZOELECTRIC POSITIONING PLATFORM}

\subsection{Structure of control model}

Since hysteresis substantially affects the stability of piezoelectric positioning system, and thereby influencing the quality of system, it is necessary to look for a robust control for the system. Sliding mode variable structure control, as a variable structure control method, features its insensitivity to external disturbance and disturbance of parameters under matching condition, as well as its simple design and easy implementation [7]. This research combines the advantages of both adaptive control and sliding mode control by applying adaptive control to sliding mode control, and uses a global adaptive sliding mode control method to achieve an accurate positioning control of the piezoelectric actuator. The structure of control system is described in Figure 3.

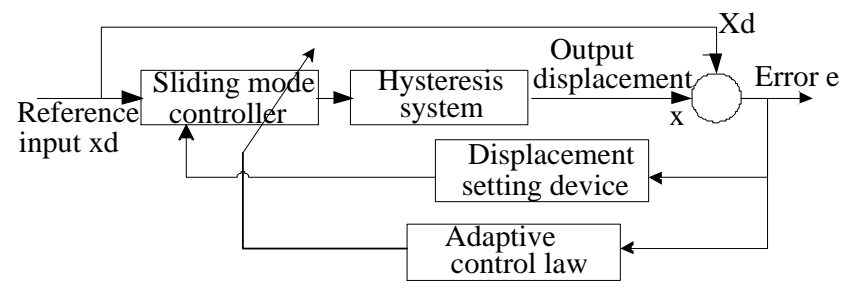

Figure 3: Structure of control system

\subsection{Controller design}

Equation (3) suggests a mathematic model for the nonlinear hysteretic control system of piezoelectric actuator. The controlled object is a secondorder system with an unknown hysteretic model. The mathematic model can be defined as

$$
\left\{\begin{array}{c}
\dot{x}_{1}=x_{2} \\
\dot{x}_{2}=-\omega_{n}^{2} x_{1}-2 \zeta \omega_{n} x_{2}+h(t)+d(t)=f(x)+b(x) u(t)+d(t)
\end{array}\right.
$$

where $h(t)$ is hysteresis output. $h(t)=P[\mathrm{U}](\mathrm{t})$ is a nonlinear model of piezoelectric stage? Functions $f(x), b(x)$ are not precisely known, and is roughly estimated as $\hat{f}(x), \hat{b}(x) . \hat{f}(x), \hat{b}(x)$ indicates the unknown external disturbance. Suppose $d(t)$ is limited and $d(t)<D . f(x)$ represents the linear model of piezoelectric stage, and the estimated value $\hat{f}(x)$ satisfies $|f(x)-\hat{f}(x)|=|\Delta f(x)| \leq F(x) . \quad 0 \leq b_{\text {min }}(x) \leq b(x) \leq b_{\text {max }}(x)$, geometric mean is selected and estimated to be $\hat{b}(x)=\sqrt{b_{\min }(x) b_{\max }(x)}$. The objective of control is to design a control law to eliminate hysteretic effects, so the state $x(t)$ follows trajectory $x_{d}(t)$, i.e. when $t \rightarrow \infty$, $x(t) \rightarrow x_{d}(t)$.

Set tracking error $e=x_{1}-x_{1 d}$, and select switching function $s=\dot{e}+m e$ (constant $m \succ 0$ ). Equation (1) satisfies the condition of sliding mode $\dot{s}=0$, and the control law is derived as follows:

$u=\frac{1}{\hat{b}(x)}\left[-\hat{f}(x)+\ddot{x}_{1 d}(x)-m \dot{e}-k \operatorname{sgn}(x)\right]$

$=u_{e q}+u_{s w}$
Where $u_{e q}=\frac{1}{\hat{b}(x)}\left(-\hat{f}(x)+\ddot{x}_{1 d}(\mathrm{t})-m \dot{e}\right)$. It is an equivalent control to compensate the known nonlinear characteristics in the system. Switching control $u_{s v}=-k \frac{1}{\hat{b}(x)} \operatorname{sgn}(s)$ compensates the uncertainties of the system, in which $k$ is switching gain. Theoretically, the discontinuous parts $u_{s w}$ in sliding control law (5) require instantaneous switching of control signal and actuator in order for the system to remain on sliding surface. However, there are unavoidable factors causing time delays in real applications. Thereby the sliding motion controlled by slide mode cannot be accurately performed on sliding surface but becomes a back-and-forth movement instead, which turns into chattering [8]. To overcome chattering phenomenon, bipolar function sigmoid is used to replace sgn ( ) , and the control law (5) is rewritten as:

$u=\frac{1}{\hat{b}(x)}\left[-\hat{f}(x)+\ddot{x}_{1 d}(x)-m \dot{e}-\eta \phi(\lambda, \mathrm{s})\right]$

where $\eta$ is switch gain. $\phi(\lambda, s)$ is bipolar function, i.e. $\phi(\lambda, s)=\frac{1-e^{-\lambda s}}{1+e^{-\lambda s}}$.In the equation, switch gain and boundary layer thickness are adjustable, so that the problems caused by excessive switch gain, constant boundary layer thickness and uncertain terms of the system can be solved. An adaptive law of parameters for switch gain and boundary layer thickness is defined as follows:

$$
\begin{aligned}
& \dot{\eta}=\gamma \hat{b}(x) \operatorname{sgn}\left(\frac{\partial s}{\partial u}\right)\left[\frac{1-e^{-\lambda s}}{1+e^{-\lambda s}}\right] s \\
& \dot{\lambda}=\varepsilon \hat{b}(x) \operatorname{sgn}\left(\frac{\partial s}{\partial u}\right) \mathrm{s}
\end{aligned}
$$

Where $\gamma, \varepsilon$, as positive real numbers, are regulation speed.

\subsection{Stability analysis}

Theorem 1 Use control laws (6) and (7) for system (4). It is validated that system (4) is stable. The chattering phenomenon and steady-state error are eliminated.

Validation: suppose function Lyapunov of system (4) is $V=\frac{1}{2} S^{2}$, then $\dot{V}=S \dot{S}=S\left[\frac{\partial S}{\partial u} \frac{\partial u}{\partial \eta} \frac{\partial \eta}{\partial t}+\frac{\partial S}{\partial u} \frac{\partial u}{\partial \lambda} \frac{\partial \lambda}{\partial t}\right]=\dot{V}_{1}+\dot{V}_{2}$

$\dot{V}_{1}=S \frac{\partial S}{\partial u} \frac{\partial u}{\partial \eta} \frac{\partial \eta}{\partial t}$

$\dot{V}_{2}=S \frac{\partial S}{\partial u} \frac{\partial u}{\partial \lambda} \frac{\partial \lambda}{\partial t}$

Plug Equation (6) into Equation (8), one obtains

$$
\begin{aligned}
& \dot{V}_{1}=S \frac{\partial S}{\partial u} \frac{\partial}{\partial \eta}\left\{\frac{1}{\hat{b}(x)}\left[\hat{f}(x)+\ddot{x}_{1 d}-m \dot{e}-\eta \phi(\lambda, S)\right]\right\} \frac{\partial \eta}{\partial t} \\
& =-S \frac{\partial S}{\partial u} \frac{1}{\hat{b}(x)} \phi(\lambda, S) \dot{\eta} \\
& =-S \frac{\partial S}{\partial u} \frac{1}{\hat{b}(x)}\left(\frac{1-e^{-\lambda S}}{1+e^{-\lambda S}}\right) \gamma \hat{b}(x) \operatorname{sgn}\left(\frac{\partial S}{\partial u}\right)\left(\frac{1-e^{-\lambda S}}{1+e^{-\lambda S}}\right) S \\
& =-\gamma\left|\frac{\partial S}{\partial u}\right|\left(\frac{1-e^{-\lambda S}}{1+e^{-\lambda S}}\right)^{2} S^{2} \leq 0
\end{aligned}
$$

Plug Equation (6) into Equation (9), one obtains 
$\dot{V}_{2}=S \frac{\partial S}{\partial u} \frac{\partial}{\partial \lambda}\left\{\frac{1}{\hat{b}(x)}\left[\hat{f}(x)+\ddot{x}_{1 d}-m \dot{e}-\eta \phi(\lambda, S)\right]\right\} \frac{\partial \lambda}{\partial t}$

$=-S \frac{\partial S}{\partial u} \frac{1}{\hat{b}(x)}\left[\eta \frac{2 S e^{-\lambda s}}{\left(1+e^{-\lambda S}\right)^{2}}\right] \dot{\lambda}$

$=-S \frac{\partial S}{\partial u} \frac{1}{\hat{b}(x)} \eta \frac{2 S e^{-\lambda s}}{\left(1+e^{-\lambda S}\right)^{2}} \varepsilon \hat{b}(x) \operatorname{sgn}\left(\frac{\partial S}{\partial u}\right)$

$=-\varepsilon \eta\left|\frac{\partial S}{\partial u}\right| \frac{2 e^{-\lambda s}}{\left(1+e^{-\lambda S}\right)^{2}} S^{2} \leq 0$

Whose solution is $\quad \dot{V}=\dot{V}_{1}+\dot{V}_{2} \leq 0$. Above all, system $V \succ 0$, only when $S=0, \dot{V}=0$, others $\dot{V} \prec 0$. Based on Lyapunov second law, system (4) is getting stable. When $t \rightarrow \infty$, error $e \rightarrow 0$, state $x_{1} \rightarrow x_{1 d}$.

\section{RESULTS AND ANALYSIS OF SIMULATION EXPERIMENT}

When model structure of piezoelectric stage is viewed as a cascade of a nonlinear hysteresis $H^{*}$ and a linear second-order system, the system can be defined as:

$$
\left\{\begin{array}{c}
\dot{x}_{1}=x_{2} \\
\dot{x}_{2}=-\omega_{n}^{2} x_{1}-2 \zeta \omega_{n} x_{2}+h(t)+d(t)=f(x)+b(x) u(t)+d(t)
\end{array}\right.
$$

Where $x_{1}=x, x_{2}=\dot{x}$, and $x$ is output displacement of piezoelectric stage. System parameters $f(x), b(x)$ are unknown. Based on prior knowledge, the approximate value is $\xi=0.43, \omega_{n}=613.4 \mathrm{rad} / \mathrm{s}$, $b(x)=800|\cos t|+23000 \quad$, disturbance term $d(t) \quad$ is limited, $d(t)=0.1 \sin (2 \pi t)$. Initial condition $x(0)=\left[\begin{array}{ll}0.5 & 0\end{array}\right]^{T}$ Reference signal $x_{d}=\sin (0.5 \pi \mathrm{t})$, select switching function $S=10 e+\dot{e}$, and take $\hat{f}(x)=-376260 x_{1}-528 x_{2}, \hat{b}(x)=22985$ 。

According to Equation (6), sliding mode control law of the controller can be defined as:

$u=\frac{1}{22985}\left[-376260 x_{1}-528 x_{2}-0.785 \sin 0.5 \pi t-10 \dot{e}-\eta \phi(\lambda, \mathrm{s})\right]$

According to Equation (7), where regulation speed $\gamma=50, \varepsilon=30$, initial value $\eta(0)=81, \lambda(0)=5.5$, and the adaptive law of parameters for switch gain and boundary layer thickness is derived as follows:

$$
\begin{aligned}
& \dot{\eta}=1149250 \operatorname{sgn}\left(\frac{\partial s}{\partial u}\right)\left[\frac{1-e^{-\lambda s}}{1+e^{-\lambda s}}\right] s \\
& \dot{\lambda}=689550 \operatorname{sgn}\left(\frac{\partial s}{\partial u}\right) \mathrm{s}
\end{aligned}
$$

The results of simulation are described in Figure 4.

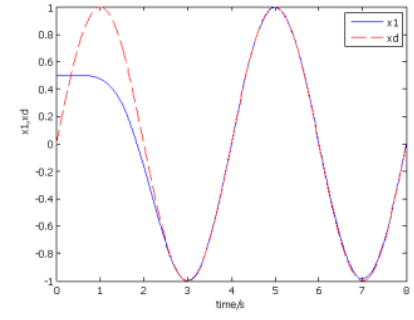

(a) Output tracking curve

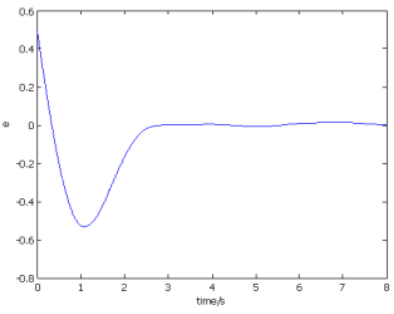

(b)Tracking error curve

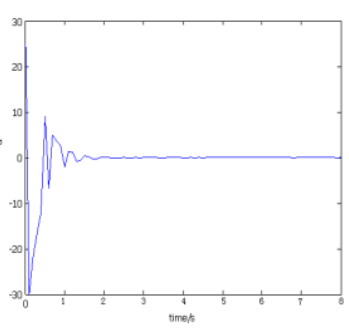

(c) Controller output curve

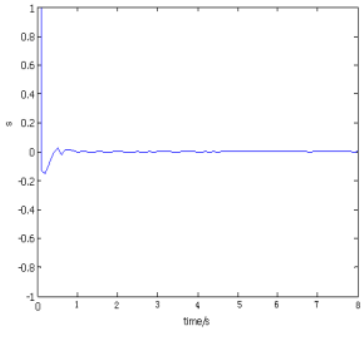

(d) Sliding surface function curve
Figure 4: Result of Simulation

The results of simulation suggest that the system tracks the expected trajectory well enough. Function curve of sliding surface goes rapidly to zero. Chattering is reduced and there is strong robustness against external disturbance. It is an effective control method for unknown system of piezoelectric stage system mode. Switch gain and boundary layer thickness can be tuned, in accordance with real-time situation, from initial value to the optimal one, since these parameters, as well as regulation speed, affect chattering and tracking accuracy and need to be adjusted repeatedly to achieve sound control.

\section{CONCLUSION}

In real-time piezoelectric actuator control system, unknown hysteretic factors substantially affect the control accuracy of system. The research introduces an adaptive sliding mode control method to cope with uncertain control issues of nonlinear system. A sliding mode strategy based on adaptive parameters of switch gain and boundary layer thickness reduces chattering and guarantees relatively high tracking accuracy. With the use of Lyapunov, the whole closed-loop system is verified as a simple and reliable control method. Simulation and experiment are conducted to validate the arithmetic effectiveness of the system.

\section{ACKNOWLEDGMENTS}

【Fund Program】Guangxi science and technology project ( Number : 2015GXNSFAA139272).

\section{REFERENCES}

[1] Kim, B., Washington, G.N., Yoon, H.S. 2012. Hysteresis-Reduced Dynamic Displacement Control of Piezoceramic Stack Actuators Using Model Predictive Sliding Mode Control [J]. Smart Materials and Structures, 21 (5): 055-018.

[2] Sun, B. 2008. Experimental Research on Dynamic Characteristic of Flexible Micro-Displacement Mechanism Based on Piezoelectric Actuator [J]. Microfabrication Technology, 1 (2), 33-36.

[3] Chen, Z., Guo, C., Zhao, J., Yuan, Z. 2006. Modeling and Control of Hysteretic Systems Based on Preisach Model [J]. Control Engineering of China, 13 (4), 270-273.

[4] Dang, X., Tan, Y. 2005. Study on Neural Networks Dynamic Hysteresis Model for Piezoceramic Based on Wiener Model [J]. Journal of System Simulation, 117 (11), 270-273.

[5] Zhao, X., Tan, Y. 2006. Modeling Perisach-Type Hysteresis Nonlinearity Using Neural Networks [J]. Control Theory and Applications, 23 (4), 581-585.

[6] Adriaens, H., Koning, W., De, Banning, R. 2000. Modeling Piezoelectric Actuators [J]. Ieee/Asme Trans. On Mechatronics, 5 (4), P331-341

[7] Zhang, Y. 2011. Adaptive Sliding Mode Control and Application For Uncertain Nonlinear Systems [D]. Chongqing University.

[8] Gao, W. 1991. Fundamentals of Variable Structure Control [M]. China Science and Technology Press. Beijing. 